\title{
Co-immunization with two recombinant Eimeria tenella lines expressing immunoprotective antigens of E. maxima elicits enhanced protection against E. maxima infection
}

Xinming Tang ${ }^{1}$, Chaoyue Wang ${ }^{2}$, Lin Liang 1,3, Dandan Hu², Sixin Zhang ${ }^{2}$, Chunhui Duan², Jingxia Suo ${ }^{2}$, Xianyong Liu ${ }^{2}$, Xun SuO ${ }^{2}$ and Shangjin Cui ${ }^{1,3^{*}}$

\begin{abstract}
Background: Live anticoccidial vaccines have been a tremendous success for disease prevention. The establishment of the reverse genetic manipulation platform has enabled the development of Eimeria parasites, the live anticoccidial vaccine strains, as vaccine vectors. In our previous study, recombinant $E$. tenella expressing a single immunodominant antigen of E. maxima (Et-EmIMP1) was able to protect chickens against challenge infection with E. maxima. This promising result encouraged us to further explore strategies to improve the protection efficacy of recombinant Eimeria and develop it as a vaccine vector.

Results: We constructed a novel recombinant Eimeria line expressing apical membrane antigen 1 of E. maxima (EtEmAMA1) and then immunized chickens with Et-EmAMA1 and/or Et-EmIMP1. We found that the E. maxima soluble antigen-specific cell-mediated immunity was much stronger in the birds that were co-immunized with Et-EmAMA1 and Et-EmIMP1 than in those that were immunized with Et-EmAMA1 or Et-EmIMP1 alone. The oocyst production after E. maxima infection was significantly reduced in the recombinant Eimeria-immunized birds compared with the wildtype-immunized and naive birds. The oocyst production in the birds co-immunized with Et-EmAMA1 and Et-EmIMP1 was consistently the lowest among the treatment groups after E. maxima infection.

Conclusions: These results demonstrated that Eimeria is an effective vaccine vector that can carry and deliver heterologous Eimeria antigens to the host immune system and trigger specific immune responses. Our results also suggested that increasing the number of recombinant Eimeria lines is an effective approach to enhance protective immunity against infections with heterologous pathogens.
\end{abstract}

Keywords: Recombinant Eimeria, Vaccine vector, Apical membrane antigen 1, Immune mapped protein 1, Immune responses

\footnotetext{
*Correspondence: cuishangjin@caas.cn

${ }^{1}$ Institute of Animal Science, Chinese Academy of Agricultural Sciences,

Beijing, China

Full list of author information is available at the end of the article
} 


\section{Background}

The genus Eimeria causes coccidiosis in a wide range of domestic and wild animals [1-5]. Eimeria parasites with good immunogenicity have been developed as virulent or attenuated live coccidiosis vaccines that have achieved great successes in practice, especially in chickens and turkeys [6,7]. With the rapid development of bioinformatics and the establishment of the genetic manipulation platform in apicomplexan parasites [8-11], Eimeria parasites have shown great potential for development as vaccine delivery vectors [12-16]. Currently, Eimeria vectors carrying immunoprotective or immunodominant antigen(s) of heterologous Eimeria species to elicit cross-protective immunities against the parental and heterologous Eimeria species is a novel strategy to develop next-generation coccidiosis vaccines. Using this strategy, one or several Eimeria species of the vaccine formulation can be removed, which can reduce the adverse pathological reaction caused by vaccination. Thus, the cost will be reduced and the safety will be improved for the novel coccidiosis vaccines $[12,16]$.

In our previous study, immune mapped protein 1 of $E$. maxima (EmIMP1) expressed by recombinant $E$. tenella was shown to be recognized by the host immune system and triggered a moderate EmIMP1-specific immune response [12]. Vaccination with this recombinant $E$. tenella line provided solid protection against $E$. tenella infection and partial protection against $E$. maxima infection in chickens [12]. These results indicate that Eimeria may be an effective antigen(s) delivery system among species with similar modes of infection and immunity. It is essential to explore strategies to improve the protection elicited by recombinant Eimeria against infections with heterologous species.

We hypothesized that the protective immunity could be strengthened by increasing the number of recombinant Eimeria lines expressing heterologous pathogens' immunodominant antigens. To investigate this hypothesis, we constructed another recombinant $E$. tenella line expressing the recognized immunoprotective antigen, apical membrane antigen 1 of E. maxima (EmAMA1) [16], and then measured its immunogenicity in this study. Moreover, we assessed the improvement of the protection of chickens after co-immunization with double recombinant Eimeria lines, i.e. a recombinant Eimeria line expressing EmAMA1 (Et-EmAMA1) constructed in this study and Et-EmIMP1 [12].

\section{Methods}

\section{Parasites and animals}

Eimeria tenella (XJ strain), E. maxima (BJ strain) and recombinant $E$. tenella expressing EmIMP1 (Et-EmIMP1) [12] were used in this project and maintained by propagating these parasites in coccidian-free, 2-5-weekold Arbor Acres (AA) broilers, which were purchased from Beijing Arbor Acres Poultry Breeding Co., Ltd. (Beijing, China). The procedures for collection, purification and sporulation were carried out as previously described [17].

One-week- or three-week-old specific pathogen-free (SPF) chickens (White Leghorn) were purchased from Merial Animal Health Co., Ltd. (Beijing, China) and were fed a pathogen-free diet and water ad libitum.

\section{Recombinant Eimeria construction}

Eimeria maxima sporozoite cDNA was synthesized as previously described [18]. Two pairs of primers were designed to mutate the Nde I restriction enzyme sites (CATATG mutated to CACATG) present in the open reading frame (ORF) of EmAMA1 (GenBank: XM_013478884.1). The whole ORF (1632 bp) was amplified with overlapping PCR using the primers AMA1F1 (5'-CAT ATG ATG TGT GGA TTG CGC GCT GC-3')/AMA1-R2 (5'-ACC GGT GTA ATC TTG GTC AAC TAA CAC G-3') from two fragments (1038 and $622 \mathrm{bp}$ ) that were amplified from the cDNA of $E$. maxima using the primers AMA1-F1/AMA1-R1 (5'-GGG GAA AAA ATA TCC ATG TGT TCC GAA G-3') and AMA1-F2 (5'-CTT CGG AAC ACA TGG ATA TTT TTT CCC C-3')/AMA1-R2, respectively (Fig. 1a). IMP1 in PSDEP2AIMP1S [12] was then replaced by Nde I and Age I restriction sites and AMA1 to generate pSDEP2AAMA1S. The transfection vector was linearized with Avr II restriction enzyme for further use.

A restriction enzyme-mediated integration (REMI) was adopted for the transfection of E. tenella sporozoites as previously described $[19,20]$. Recombinant E. tenella line, Et-EmAMA1, was selected by fluorescence-activated cell sorting combined with drug pressure (Table 1). The feed was mixed with pyrimethamine (CAS:58-4-0; J\&K Scientific Ltd., Beijing, China) by successive serial dilutions, and the final concentration was $150 \mathrm{mg} / \mathrm{kg}$ feed. After several generations of selection, PCR was conducted using the AMA1-F1 and AMA1-R2 primers from genomic DNA of recombinant Eimeria to confirm the insertion of the exogenous EmAMA1 gene. Western blot and indirect immunofluorescent assay (IFA) were conducted to confirm foreign antigen expression and distribution in recombinant parasites based on the previously described protocols, respectively $[18,21]$. Recombinant Eimeria parasite soluble antigens were resolved by SDS-PAGE and immunoblot analysis following standard protocols with mouse anti-flag monoclonal antibody and HRP-conjugated goat anti-mouse IgG (Proteintech, Chicago, IL, USA) as primary and secondary antibodies. Poly-antibodies against $E$. tenella GAPDH served as the 


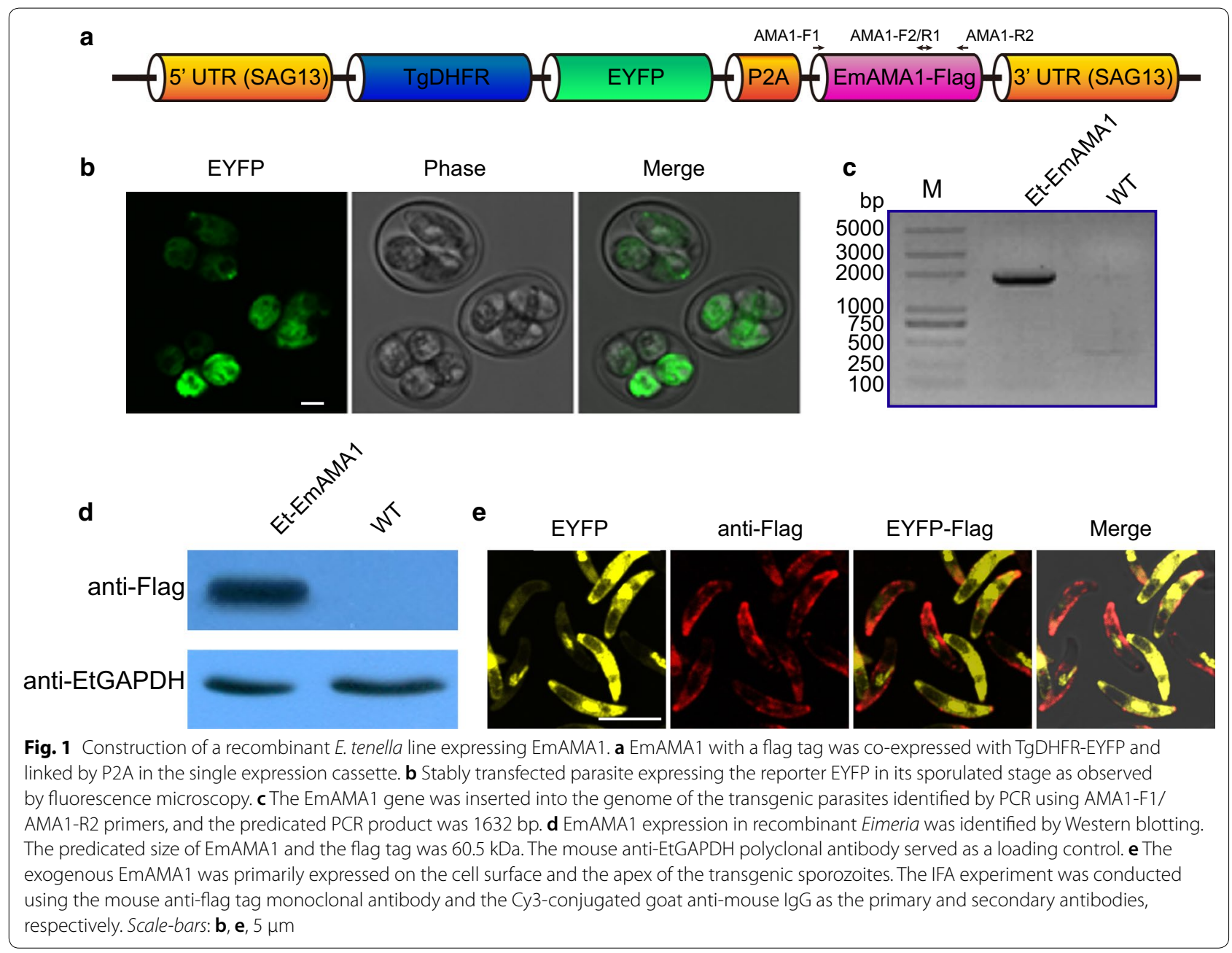

Table 1 Et-EmAMA1 selection based on EYFP expression

\begin{tabular}{lll}
\hline Generations & \% EYFP expression & Selection strategy \\
\hline 1 & 32.3 & Drug \\
2 & 78.3 & Drug +FACS \\
3 & 93.2 & Drug $^{\text {a FACS }}$ \\
4 & 96.5 & Drug $^{\text {b }}$ \\
5 & 94.7 & Drug $^{-3}$ \\
6 & 94.2 & c $^{-}$ \\
\hline
\end{tabular}

${ }^{a}$ Facilitating recombinant Eimeria parasite selection by pyrimethamine combined with fluorescence-activated cell sorting (FACS)

b Stabilization of the recombinant Eimeria population by pyrimethamine

c The recombinant Eimeria population was stable without selection pressure

loading control. Indirect immunofluorescent assays were conducted to detect the distribution of EmAMA1 in the sporozoite stage of the recombinant Eimeria by mouse anti-flag monoclonal antibody and Cy3-conjugated goat anti-mouse IgG (Proteintech).

\section{ELISA and ELISPOT}

Five groups of three-week-old SPF chickens (6 chickens/ group) were either left naïve (Ctrl) or were immunized by infection with 200 sporulated wild-type E. tenella (WT), Et-EmIMP1, Et-EmAMA1 and an equivalent mixture of Et-EmIMP1 and Et-EmAMA1 oocysts. Secondary immunization was administered at 2-week intervals with 5000 oocysts as the immunization dosage for each bird. The $E$. maxima oocysts antigen (EmAg)-specific humoral and cellular immune responses were analyzed by ELISA and enzyme-linked immunospot assay (ELISPOT), respectively. EmAg was obtained from purified sporulated oocysts as previously described [22]. EmAg $(5 \mu \mathrm{g} / \mathrm{ml})$ was coated onto the individual wells of the plate followed by a reaction with the serum (diluted in 1:100) collected from the birds at 2 weeks after primary and secondary (booster) immunization. The HRP-conjugated goat antichicken IgY Fc fragment (diluted in 1:5000; Bethyl Laboratories, Inc., Montgomery, TX, USA) was used as the secondary antibody. The optical density was measured by 
a microplate reader (Model 60; Bio-Rad, Hercules, CA, USA) at $450 \mathrm{~nm}$.

Eimeria maxima parasite antigen-specific cellular immune responses revealed by IFN $-\gamma$ secreting cells present in peripheral blood mononuclear cells (PBMCs) were evaluated byELISPOT at 2 weeks after secondary immunization following the established protocols [21, 23]. Briefly, $1 \times 10^{6}$ PBMCs from the 3 randomly selected birds of each group were separately stimulated with $10 \mu \mathrm{l}$ of PBS, $10 \mu \mathrm{l}$ of E. maxima oocysts antigen (EmAg, 10 $\mu \mathrm{g} / \mathrm{ml}$ ) or $10 \mu \mathrm{l}$ Phorbol-12-myristate-13-acetate (PMA) plus ionomycin (10 $\mathrm{ng} / \mathrm{ml}$ PMA plus $5 \mu \mathrm{g} / \mathrm{ml}$ ionomycin). IFN- $\gamma$-secreting lymphocytes were detected after $24 \mathrm{~h}$ of stimulation.

\section{Vaccination and challenge infection}

Groups of inbred SPF chickens (18 chickens/group) were either left naïve (Ctrl) or were immunized by infection with 200 sporulated wild-type E. tenella (WT) oocysts, 200 sporulated Et-EmIMP1 oocysts, 200 sporulated Et-EmAMA1 oocysts and 100 sporulated Et-EmIMP1 together with 100 sporulated Et-EmAMA1 oocysts at one week of age. New chopped straw litter was spread over the bottom of the cages to a depth of $5 \mathrm{~cm}$. The chickens were housed under the same temperature and humidity conditions and fed a coccidian-free diet and water $a d$ libitum. Six chickens from each group were separately removed to new cages (which contained a metal mesh which separated the chickens from feces) at 14, 21 and 42 dpi. The chickens were orally challenged with E. maxima (50 oocysts/bird) after each removal. The fecal samples were collected every day from day 5 to 12 after each challenge infection. The total number of oocysts in the feces was evaluated using a McMaster egg counting chamber after each challenge infection.

\section{Statistical analysis}

GraphPad Prism v.6.01 (GraphPad Software) was used for statistical analysis. Differences in experimental treatments were tested using Duncan's Multiple Range Test following ANOVA with significance reported at $P \leq 0.05$.

\section{Results}

\section{Construction of a recombinant $E$. tenella line expressing $E$. maxima AMA1 (Et-EmAMA1)}

In the last decade, a technical platform for the construction and selection of recombinant Eimeria parasites has been successfully developed $[9,20]$. In this study, the transfection plasmid, pSDEP2AAMA1S (Fig. 1a) was constructed based on the previously constructed pSDEP2AIMP1S plasmid [12]. This new plasmid contains a single expression cassette in which the selected marker gene and EmAMA1 gene tagged with the flag epitope were linked by a P2A sequence, which mediates the cleavage of the dual flanking proteins [24]. The expression cassette was controlled by the EtSAG13 (E. tenella surface antigen 13) promoter (Fig. 1a) [25]. Eimeria tenella sporozoites were transfected with the linearized pSDEP2AAMA1S plasmid and then inoculated into the cloacal opening of chicks. The percentage of reporter positive recombinant Eimeria (Fig. 1b) in the 1st generation progeny was $32.3 \%$ (Table 1 ). The positive population of recombinant Eimeria tended to be stable after 3 generations under selection pressure (Table 1). In addition, the recombinant population remained stable without selection pressure (Table 1).

Next, we conducted serial experiments to identify the expression of exogenous EmAMA1 in the recombinant parasites. We confirmed the insertion of the EmAMA1 gene into the recombinant Eimeria genome by PCR using specific primers (Fig. 1c). The expression of EmAMA1 in the sporulated oocyst stage was demonstrated by Western botting using flag-tag specific-antibodies (Fig. 1d). We found that exogenous EmAMA1 was mainly expressed on the cell surface and the apex of the recombinant Eimeria sporozoites (Fig. 1e). These data showed that we obtained a recombinant Eimeria population stably expressing exogenous EmAMA1.

\section{Et-EmAMA1 elicited E. maxima antigen specific cellular immunity}

In our previous study, we demonstrated that exogenous antigen, i.e. EmIMP1, expressed by Eimeria efficiently elicited EmIMP1 and E. maxima antigen-specific immune responses [12]. In this study, we detected the E. maxima antigen (EmAg)-specific immune responses elicited by Et-EmAMA1 and then focused on detecting whether immunity was enhanced by co-immunization with Et-EmAMA1 and Et-EmIMP1.We analyzed the EmAg-specific antibody production in the recombinant Eimeria-immunized birds and found that EmAg-specific antibody production was not significantly increased after co-immunization with Et-EmAMA1 and Et-EmIMP1 compared with immunization with Et-EmAMA1 or EtEmIMP1 alone (Fig. 2a). We detected EmAg-specific cellular immunity after booster immunization with the single or double recombinant Eimeria lines, as revealed by measuring the number of IFN- $\gamma$-secreting lymphocytes in PBMCs (Fig. 2b, c). The EmAg-specific cellular immunity was shown to be significantly improved after the co-immunization with Et-EmAMA1 and Et-EmIMP1 compared to the immunization with Et-EmAMA1 or Et-EmIMP1 alone (Fig. 2b, c). The enhanced immune responses elicited by co-immunization with the double recombinant Eimeria lines suggest that the protection 
a

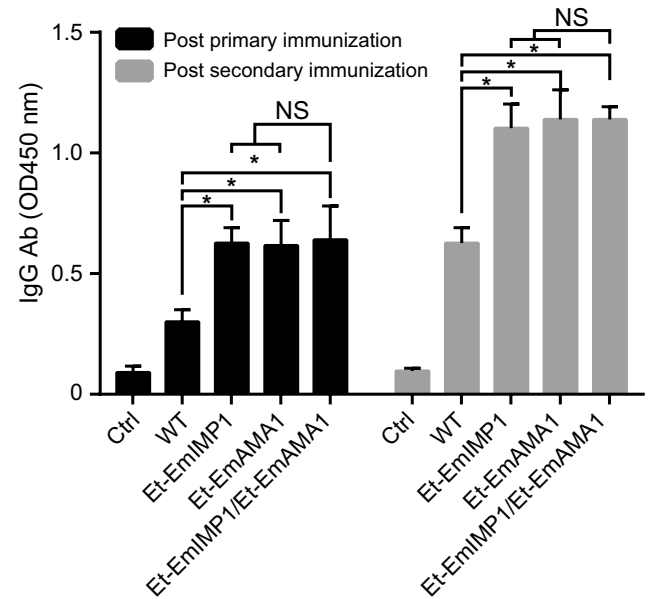

b

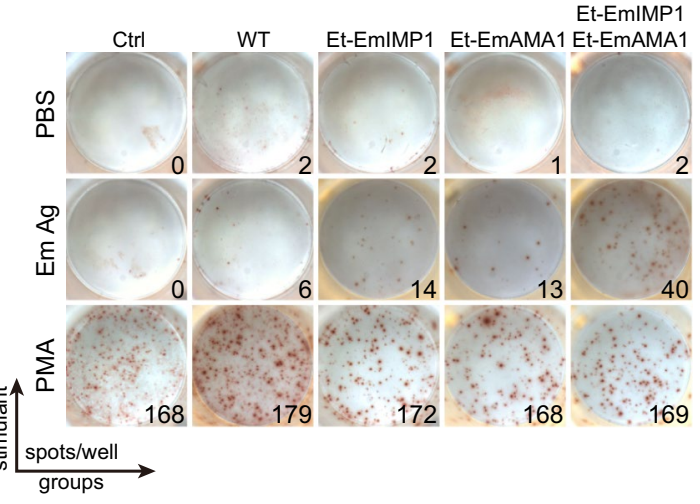

c

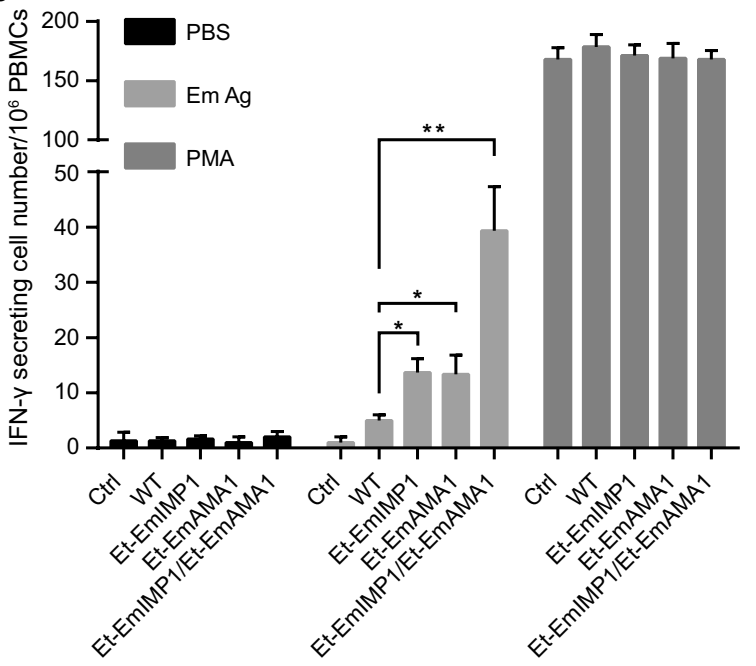

Fig. 2 Immunization with Et-EmAMA1 and/or Et-EmIMP1 elicited E. maxima-specific humoral and cell-mediated immunity. a The EmAg-specific antibody production after primary and secondary immunization with recombinant Eimeria line(s) or wild-type parasites. b, c The EmAg-specific cellular immune responses after secondary immunization was analyzed by ELISPOT. The representative (b) and mean number (c) of IFN- $\gamma$-secreting lymphocytes (spots) in PBMCs from naïve (Ctrl), wild-type E. tenella (WT), Et-EmAMA1 only, Et-EmIMP1 only, and Et-EmAMA1 and Et-EmIMP1 immunized birds after stimulation with PBS, E. maxima oocyst antigens (Em Ag) and PMA plus ionomycin (PMA+lon), $(n=3)$. ${ }^{*} P<0.05,{ }^{* *} P<0.01$. Abbreviation: NS, no significant difference 
against E. maxima is much better than immunization with the single recombinant Eimeria line.

\section{Co-immunization with Et-EmAMA1 and Et-EmIMP1 enhanced the protective efficacy against E. maxima infection}

To test whether the protective efficacy against E. maxima infection by immunization with double recombinant Eimeria lines is higher than that by immunization with a single recombinant Eimeria line, the birds were challenged with a low dose of E. maxima oocysts at different time points. We found that the oocyst output after challenge infection was significantly reduced in birds immunized with either Et-EmAMA1 or Et-EmIMP1 compared with the wild-type-immunized birds (Fig. 3). We also found that the birds immunized with Et-EmAMA1 and Et-EmIMP1 produced nearly half the number of oocysts compared to Et-EmAMA1- or Et-EmIMP1-immunized birds after challenge with E. maxima at 14 dpi (Fig. 3a), $21 \mathrm{dpi}$ (Fig. 3b) or 42 dpi (Fig. 3c). These results demonstrated that the protective efficacy was much higher with the double recombinant Eimeria lines than the same dosage immunization with a single recombinant Eimeria line (Fig. 3). In addition, the protection against E. maxima infection was established as early as $14 \mathrm{dpi}$ (Fig. 3a). The number of the oocyst was gradually decreased after challenge infection at $14 \mathrm{dpi}$ (Fig. 3a), $21 \mathrm{dpi}$ (Fig. 3b) and 42 dpi (Fig. 3c). The above results demonstrate that recombinant Eimeria triggered a heterologous pathogenspecific immunity and partly protected chickens against low-dose heterologous pathogen infection.

\section{Discussion}

In this study, we successfully obtained a recombinant $E$. tenella line expressing heterologous EmAMA1 and tested its immunogenicity. We found that both Et-EmAMA1 and Et-EmIMP1 [12] could protect chickens against heterologous E. maxima infection, and the protection could be enhanced by co-immunization with double recombinant Eimeria lines. In addition to previous findings, these results suggested that Eimeria is an effective vaccine vector that can carry and deliver Eimeria and other pathogens' antigens to the host immune system and trigger specific immune responses [12-15]. Our results also suggest that increasing the number of recombinant Eimeria lines may be an effective approach to strengthen the protective immunity against infections with heterologous pathogens. This is of great significance for developing next-generation coccidiosis vaccines that can simplify formulations of recombinant vaccines for animals and humans.

Our results show that recombinant Eimeria efficiently elicited heterologous antigen specific antibody production and cell-mediated immunity. The cell-mediated immunity rather than antibody production was improved when co-immunized with double recombinant Eimeria lines (Fig. 2) suggest that the enhanced protection by co-immunization is related to cellular immunity. Parallel experiments demonstrated that the secretion or cell surface display of EmAMA1 in recombinant E. tenella elicited EmAMA1 specific-antibody production. Multiple immunizations with recombinant Eimeria could partly protect the chickens against a moderate dose $(300$ oocysts/bird) of E. maxima infection [16]. The reduced oocyst production after challenge infection in recombinant Eimeria immunized birds may be related to the
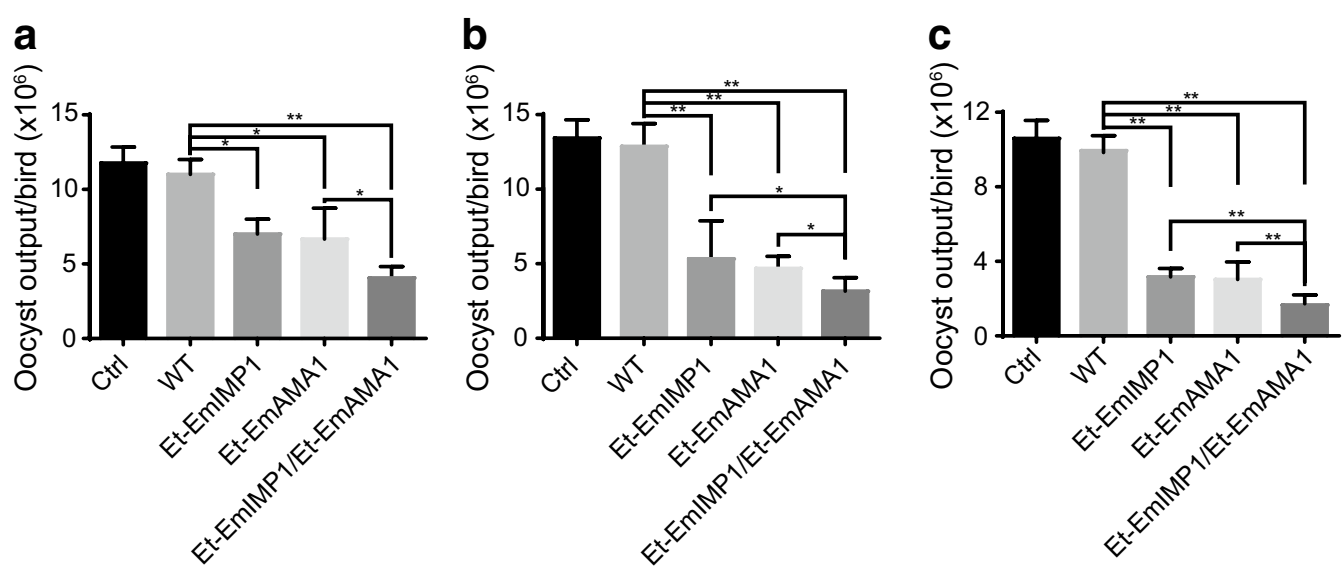

Fig. 3 Protection against E. maxima infection provided by co-immunization with Et-EmAMA1 and Et-EmIMP1. a-c Oocyst output after challenge with E. maxima in the chickens immunized with or without Et-EmIMP1 and/or Et-EmAMA1 or its wild-type at 14 (a), 21 (b) and $42 \mathrm{dpi}(\mathbf{c})$. ${ }^{*} P<0.05$, ${ }^{* *} P<0.01$ 
reduced serum IL-10 level [16]. Using EmIMP1 in recombinant protein or EmAMA1 in DNA formulations has been reported to achieve a reduction of approximately $45 \%$ in parasite production in small-scale vaccination trials $[26,27]$. The reduction in oocyst production was $30-90 \%$ using E. tenella or E. acervulina antigens in recombinant protein, DNA or live-vector formulations with or without cytokines as adjuvants [27, 28]. Moreover, the protection against E. tenella, E. necatrix, E. maxima or E. acervulina infection was also reported to be enhanced using multivalent epitope DNA vaccines from multiple antigens compared to a single antigen [2934]. Although those subunit or recombinant vaccines achieved promising successes in the laboratory and are much safer and less expensive than live anticoccidial vaccines, no large-scale trials have been reported [27]. One explanation may be the lack of automation systems for mass immunization and that chickens experience more stress with intramuscular injection of recombinant protein and DNA vaccines than with oral immunization with live anticoccidial vaccines [35-37]. Another explanation could be that the achieved protection through recombinant protein and DNA vaccines can only be detected after one or two booster immunizations, whereas a single oral immunization with live oocysts can elicit long-term protective immunity $[6,38]$.

To date, no matter the form of antigen delivery, i.e. recombinant protein, DNA or live vector including Eimeria, antigens can elicit complete protection as effective as live anticoccidial vaccines in the field. Our present results show promise for improving immune protection by recombinant Eimeria by increasing the number or category of heterologous antigens. Solutions include but are not limited to: (i) using advanced biotechnological tools to discover new immunodominant antigen(s) of Eimeria, such as IMP1 [26]; (ii) improving the heterologous antigen expression level by using a stronger promoter or increasing the gene copy number, etc. [24]; (iii) coexpressing multiple immunodominant antigens mediated by P2A in one recombinant Eimeria line or by double or multiple expression cassette [24, 39]; (iv) optimizing the antigens' location in recombinant Eimeria for efficient recognition by host immune system [18]; and (v) fusion expression of heterologous antigens with cytokine(s) or other molecular adjuvants $[21,25,40]$.

\section{Conclusions}

Recombinant E. tenella expressing an immunodominant antigen of E. maxima (EmAMA1) elicited partial protection against E. maxima infection in chickens, and the protection was improved by co-immunization with two recombinant Eimeria lines (Et-EmIMP1 and
Et-EmAMA1). Our results provide a good basis for further development of next-generations coccidiosis vaccines and possess great significance for developing other recombinant vaccines for animals and humans.

\section{Abbreviations \\ IMP1: immune mapped protein 1; AMA1: apical membrane antigen 1; SAG13: surface antigen 13; EmAg: Eimeria maxima antigen; PBMCs: peripheral blood mononuclear cells; dpi: days post-immunization; dpc: days post-challenge; ORF: open reading frame; REMl: restriction enzyme-mediated integration; IFA: indirect immunofluorescent assay; SPF: specific pathogen free; ELISPOT: enzyme-linked immunospot assay; PMA: phorbol-12-myristate-13-acetate.}

\section{Acknowledgments}

Not applicable.

\section{Authors' contributions}

XT, XS and SC conceived and designed this study and analyzed the data. XT carried out the experiments and drafted the manuscript. CW, LL, DH, SZ, CD and JS contributed to conducting animal experiments and the statistical analysis. XL, XS and SC supervised the study implementation and revised the manuscript. All authors read and approved the final manuscript.

\section{Funding}

This work was supported by the Agricultural Science and Technology Innovation Program of China (ASTIP-IAS15), the National Natural Science Foundation of China (31472180) and the China Postdoctoral Science Foundation (2018M641566).

\section{Availability of data and materials}

All data generated or analyzed during this study are included in this published article.

\section{Ethics approval and consent to participate}

Animal experiments were approved by the Beijing Administration Committee of Laboratory Animals. All animal experiments were performed in strict accordance with the China Agricultural University Institutional Animal Care and Use Committee guidelines (CAU20160629-1) and followed the International Guiding Principles for Biomedical Research Involving Animals.

\section{Consent for publication}

Not applicable.

\section{Competing interests}

The authors declare that they have no competing interests.

\section{Author details \\ ${ }^{1}$ Institute of Animal Science, Chinese Academy of Agricultural Sciences, Bei- jing, China. ${ }^{2}$ Key Laboratory of Zoonosis of Ministry of Agriculture \& National Animal Protozoa Laboratory, College of Veterinary Medicine, China Agricultural University, Beijing, China. ${ }^{3}$ Beijing Scientific Observation and Experimental Station of Veterinary Drugs and Diagnostic Technology, Ministry of Agriculture, Beijing, China.}

Received: 9 January 2019 Accepted: 6 July 2019

Published online: 12 July 2019

\section{References}

1. Chapman HD, Barta JR, Blake D, Gruber A, Jenkins M, Smith NC, et al. A selective review of advances in coccidiosis research. Adv Parasitol. 2013;83:93-171.

2. Hillman AE, Yang R, Lymbery AJ, Thompson RCA. Eimeria spp infecting quenda (Isoodon obesulus) in the greater Perth region, Western Australia. Exp Parasitol. 2016;170:148-55. 
3. Leclaire S, Faulkner CT. Gastrointestinal parasites in relation to host traits and group factors in wild meerkats Suricata suricatta. Parasitology. 2014:141:925-33.

4. Paul GC, Friend DG. Clostridial enterotoxemia and coccidiosis in weanling cottontail rabbits (Sy/vilagus audubonii, Sylvilagus floridanus, Sylvilagus nuttallii) from Colorado, USA. J Wildl Dis. 2018. https://doi. org/10.7589/2018-02-026.

5. Shirley MW. Eimeria spp. from the chicken: occurrence, identification and genetics. Acta Vet Hung. 1997:45:331-47.

6. Williams RB. Fifty years of anticoccidial vaccines for poultry (1952-2002). Avian Dis. 2002;46:775-802

7. Chapman HD. Coccidiosis in the turkey. Avian Pathol. 2008;37:205-23.

8. Hanig S, Entzeroth R, Kurth M. Chimeric fluorescent reporter as a tool for generation of transgenic Eimeria (Apicomplexa, Coccidia) strains with stage specific reporter gene expression. Parasitol Int. 2012;61:391-8.

9. Yan W, Liu X, Shi T, Hao L, Tomley FM, Suo X. Stable transfection of Eimeria tenella: constitutive expression of the YFP-YFP molecule throughout the life cycle. Int J Parasitol. 2009;39:109-17.

10. Kelleher M, Tomley FM. Transient expression of beta-galactosidase in differentiating sporozoites of Eimeria tenella. Mol Biochem Parasitol. 1998:97:21-31.

11. Hao LL, Liu XY, Zhou XY, Li JD, Suo X. Transient transfection of Eimeria tenella using yellow or red fluorescent protein as a marker. Mol Biochem Parasit. 2007;153:213-5.

12. Tang X, Liu X, Yin G, Suo J, Tao G, Zhang S, et al. A novel vaccine delivery model of the apicomplexan Eimeria tenella expressing Eimeria maxima antigen protects chickens against infection of the two parasites. Front Immunol. 2017;8:1982.

13. Tang X, Yin G, Qin M, Tao G, Suo J, Liu X, et al. Transgenic Eimeria tenella as a vaccine vehicle: expressing TgSAG1 elicits protective immunity against Toxoplasma gondii infections in chickens and mice. Sci Rep. 2016:6:29379.

14. Clark JD, Oakes RD, Redhead K, Crouch CF, Francis MJ, Tomley FM, et al. Eimeria species parasites as novel vaccine delivery vectors: anti-Campylobacter jejuni protective immunity induced by Eimeria tenella-delivered CjaA. Vaccine. 2012;30:2683-8.

15. Marugan-Hernandez V, Cockle C, Macdonald S, Pegg E, Crouch C, Blake DP, Tomley FM. Viral proteins expressed in the protozoan parasite Eimeria tenella are detected by the chicken immune system. Parasit Vectors. 2016;9:463.

16. Pastor-Fernandez I, Kim S, Billington K, Bumstead J, Marugan-Hernandez $\checkmark$, Kuster T, et al. Development of cross-protective Eimeria-vectored vaccines based on apical membrane antigens. Int J Parasitol. 2018;48:505-18.

17. Long PL, Millard BJ, Joyner LP, Norton CC. A guide to laboratory techniques used in the study and diagnosis of avian coccidiosis. Folia Vet Lat. 1976;6:201-17.

18. Huang $X$, Zou J, Xu H, Ding Y, Yin G, Liu X, et al. Transgenic Eimeria tenella expressing enhanced yellow fluorescent protein targeted to different cellular compartments stimulated dichotomic immune responses in chickens. J Immunol. 2011;187:3595-602.

19. Liu X, Shi T, Ren H, Su H, Yan W, Suo X. Restriction enzyme-mediated transfection improved transfection efficiency in vitro in apicomplexan parasite Eimeria tenella. Mol Biochem Parasitol. 2008;161:72-5.

20. Clark JD, Billington K, Bumstead JM, Oakes RD, Soon PE, Sopp P, et al. A toolbox facilitating stable transfection of Eimeria species. Mol Biochem Parasitol. 2008;162:77-86.

21. Li Z, Tang X, Suo J, Qin M, Yin G, Liu X, et al. Transgenic Eimeria mitis expressing chicken interleukin 2 stimulated higher cellular immune response in chickens compared with the wild-type parasites. Front Microbiol. 2015;6:533.

22. de Venevelles P, Chich JF, Faigle W, Loew D, Labbe M, Girard-Misguich F, et al. Towards a reference map of Eimeria tenella sporozoite proteins by two-dimensional electrophoresis and mass spectrometry. Int J Parasitol. 2004;34:1321-31.
23. Yin G, Qin M, Liu X, Suo J, Suo X. Interferon-gamma enzyme-linked immunosorbent spot assay as a tool to study $T$ cell responses to Eimeria tenella infection in chickens. Poult Sci. 2013:92:1758-63.

24. Tang X, Liu X, Tao G, Qin M, Yin G, Suo J, et al. "Self-cleaving" 2A peptide from porcine teschovirus-1 mediates cleavage of dual fluorescent proteins in transgenic Eimeria tenella. Vet Res. 2016;47:68.

25. Tang X, Suo J, Li C, Du M, Wang C, Hu D, et al. Transgenic Eimeria tenella expressing profilin of Eimeria maxima elicits enhanced protective immunity and alters gut microbiome of chickens. Infect Immun. 2018;86:e00888.

26. Blake DP, Billington KJ, Copestake SL, Oakes RD, Quail MA, Wan KL, et al. Genetic mapping identifies novel highly protective antigens for an apicomplexan parasite. PLoS Pathog. 2011;7:e1001279.

27. Blake DP, Pastor-Fernandez I, Nolan MJ, Tomley FM. Recombinant anticoccidial vaccines-a cup half full? Infect Genet Evol. 2017;55:358-65.

28. Wang Q, Chen L, Li J, Zheng J, Cai N, Gong P, et al. A novel recombinant BCG vaccine encoding Eimeria tenella rhomboid and chicken IL-2 induces protective immunity against coccidiosis. Korean J Parasitol. 2014;52:251-6.

29. Liu T, Huang J, Li Y, Ehsan M, Wang S, Zhou Z, et al. Molecular characterisation and the protective immunity evaluation of Eimeria maxima surface antigen gene. Parasit Vectors. 2018;11:325.

30. Song X, Gao Y, Xu L, Yan R, Li X. Partial protection against four species of chicken coccidia induced by multivalent subunit vaccine. Vet Parasitol. 2015;212:80-5.

31. Song $X, X u L$, Yan R, Huang $X$, Li X. Construction of Eimeria tenella multiepitope DNA vaccines and their protective efficacies against experimental infection. Vet Immunol Immunopathol. 2015;166:79-87.

32. Song X, Zhang Z, Liu C, Xu L, Yan R, Li X. Evaluation of the persistence, integration, histopathology and environmental release of DNA vaccine encoding Eimeria tenella TA4 and chicken IL-2. Vet Parasitol. 2016;229:22-30.

33. Song $X$, Zhao $X, X u L$, Yan $R$, Li X. Immune protection duration and efficacy stability of DNA vaccine encoding Eimeria tenella TA4 and chicken IL-2 against coccidiosis. Res Vet Sci. 2017;111:31-5.

34. Du A, Wang S. Efficacy of a DNA vaccine delivered in attenuated Salmonella typhimurium against Eimeria tenella infection in chickens. Int J Parasitol. 2005;35:777-85.

35. Del Cacho E, Gallego M, Lillehoj HS, Quilez J, Lillehoj EP, Sanchez-Acedo C. Induction of protective immunity against experimental Eimeria tenella infection using serum exosomes. Vet Parasitol. 2016;224:1-6.

36. Song $X, X u L$, Yan $R$, Huang $X$, Shah MA, Li X. The optimal immunization procedure of DNA vaccine PCDNA-TA4-IL-2 of Eimeria tenella and its cross-immunity to Eimeria necatrix and Eimeria acervulina. Vet Parasitol. 2009;159:30-6

37. Yin G, Lin Q, Qiu J, Qin M, Tang X, Suo X, et al. Immunogenicity and protective efficacy of an Eimeria vaccine candidate based on Eimeria tenella immune mapped protein 1 and chicken CD40 ligand. Vet Parasitol. 2015;210:19-24.

38. Shirley MW, Smith AL, Tomley FM. The biology of avian Eimeria with an emphasis on their control by vaccination. Adv Parasitol. 2005;60:285-330.

39. Yin G, Liu X, Zou J, Huang X, Suo X. Co-expression of reporter genes in the widespread pathogen Eimeria tenella using a double-cassette expression vector strategy. Int J Parasitol. 2011;41:813-6.

40. Qin M, Tang X, Yin G, Liu X, Suo J, Tao G, et al. Chicken IgY Fc expressed by Eimeria mitis enhances the immunogenicity of $E$. mitis. Parasit Vectors. 2016;9:164.

\section{Publisher's Note}

Springer Nature remains neutral with regard to jurisdictional claims in published maps and institutional affiliations. 